\title{
ARCHIVOS Y DOCUMENTOS DE LOS ANTIGUOS BANCOS DE EMISIÓN EXISTENTES EN EL ARCHIVO GENERAL DE LA NACIÓN
}

\author{
Leonor Ludlow*
}

1 1 fondo de los Antiguos Bancos de Emisión se localiza en la galería 2 del Archivo General de la Nación (AGN), en éste se conservan libros y expedientes de varias de las instituciones de emisión, que formaron parte del primer régimen bancario mexicano. Suman un total de 3150 volúmenes correspondientes a 17 de las 30 instituciones de crédito que tuvieron vigencia entre 1882 y $1932 .^{1}$

Para su consulta, se cuenta con un inventario general. Hace algunos años con el apoyo financiero de diversas instituciones se catalogaron varios archivos de este fondo, bajo mi supervisión y contando para ello con el trabajo y conocimiento del personal del AGN. ${ }^{2}$ A fin de facilitar su consulta se publicaron las guías de los bancos Mercantil de Veracruz, y de la asociación bancaria que formaron los bancos de Chiapas y de Oaxaca fusionados con el Oriental de México. ${ }^{3}$ También han sido catalogados los archivos de Aguascalientes, Guanajuato y Querétaro, pero no han sido publicados. ${ }^{4}$ Queda pendiente por ordenar y clasificar otros diez establecimientos. ${ }^{5}$

* Instituto de Investigaciones Históricas-uNAM.

${ }^{1}$ Archivo, Guía, 1990, p. 327. La guía se puede consultar en Internet: \http://www.agn.gob.mx/ indice.html .

${ }^{2}$ Se recibió financiamiento de CONACYT, además del apoyo de diversas instituciones académicas como fueron el Instituto Mora, el Instituto de Investigaciones Históricas y la Universidad Veracruzana. María Teresa Pacho, Carlos Contreras y Noé Rojas Palma ejecutaron estas tareas.

${ }^{3}$ Ludlow y Blázquez, Catálogo, 1997; Ludlow, Catálogo, 2001.

${ }^{4}$ El apoyo financiero para esta catalogación fue otorgado por El Colegio de Michoacán.

${ }^{5}$ Están en espera de catalogación los fondos correspondientes a los bancos de Coahuila, Comercial Refaccionario de Chihuahua, de Durango, de Guerrero, Minero de Chihuahua, Comercial de Chihuahua, Mexicano de Chihuahua, de San Luis Potosí y de Tabasco. 
Antes de revisar los contenidos de este grupo documental, descrito en el cuadro 1, haré una breve revisión histórica de estas instituciones de crédito, con el objeto de facilitar al lector la comprensión de este escrito.

La banca moderna en México nació en 1864, con la fundación del primer banco comercial, el Bank of London, Mexico and Southamerica, sucursal de una red londinense con representaciones en diversas regiones del mundo. Dos décadas más tarde, dio inicio la primera fase de fundaciones bancarias, con la apertura de varias empresas bancarias en el estado de Chihuahua (Santa Eulalia, Comercial de Chihuahua, Hidalgo del Parral), así como de los bancos Nacional Mexicano y Mercantil Mexicano en la ciudad de México, los cuales se fusionaron bajo la denominación de Banco Nacional de México. Durante los años siguientes se fundaron diversos establecimientos bancarios en aquellas entidades con mayor dinamismo agrícola y minero, fundamentalmente volcado a la exportación, como fue el caso de los estados de Durango, Nuevo León, Zacatecas y Yucatán. ${ }^{6}$

Dichas empresas carecieron de un ordenamiento legal que regulara sus actividades, ya que el capítulo de "Bancos" del Código de Comercio de 1884 fue cancelado dos años después. La legislación bancaria se retrasó hasta 1897, fecha de emisión de la primera Ley de Instituciones de Crédito del país, gracias a la cual se multiplicaron el número de establecimientos de emisión, circulación y descuento, con casa matriz en la mayoría de estados del país, además de la red de agencias y corresponsales en diversas plazas mercantiles en el propio estado, como se aprecia en el cuadro 2 en el que se enlista, en orden alfabético, el nombre de la institución y la fecha de fundación.

La multiplicación de instituciones de crédito a principios del siglo $\mathrm{xx}$ expandió los servicios bancarios hacia numerosos rincones del país, gracias a las actividades de esos bancos estatales que, además de las funciones comerciales (depósito y circulación) que fueron propias de esos establecimientos, cumplieron con la tarea de emitir, y con ello dotaron a los mercados locales de medios de pago confiables y uniformes, facilitando así las transacciones mercantiles.

${ }^{6}$ Véase Ludlow, "Primera", 1993, pp. 330-362.

${ }^{7}$ Werner Sombart, afirmó que en sus inicios este tipo de bancos se confundió con los bancos de depósito, pero "por su honorabilidad y prestigio, llega a ser un regulador de la vida económica y sus cifras de negocios reflejan el curso que aquélla sigue", lo que se manifestó tanto por crecimiento en el volumen de las emisiones como en la expansión en la circulación de sus billetes; Sombart, Apogeo, 1984, vol. I, pp. 76-79. Véase Ludlow, "Ejemplos", 2003. 


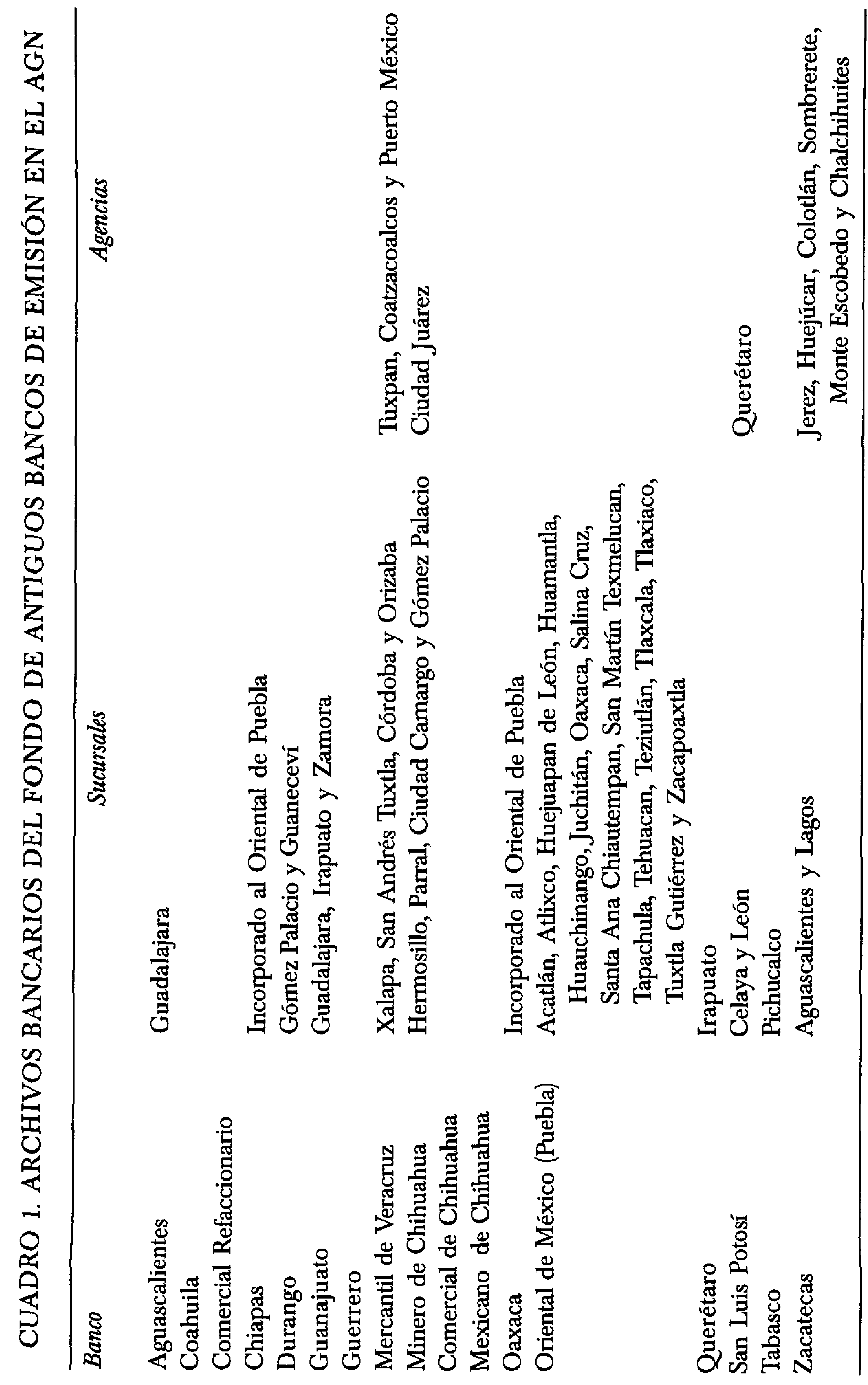


CUADRO 2. FUNDACIÓN DE INSTITUCIONES BANCARIAS

Institución

Año

Banco de Aguascalientes 1902

Banco de Campeche 1903

Banco de Coahuila 1897

Banco de Chiapas 1899

Banco de Guanajuato 1900

Banco de Guerrero 1906

Banco de Hidalgo 1902

Banco de Jalisco 1898

Banco de Michoacán 1902

Banco de Morelos 1903

Banco de Sonora 1898

Banco del Estado de México 1897

Banco Mercantil de Monterrey 1898

Banco Mercantil de Veracruz 1898

Banco Occidental de México, Sinaloa 1898

Banco Oriental de México, Puebla 1900

Banco de Oaxaca 1899

Banco de Querétaro 1903

Banco de San Luis Potosí 1898

Banco de Tabasco 1901

Banco de Tamaulipas 1902

El sistema bancario mexicano de fines del siglo XIX se asemeja a otras experiencias nacionales que, en periodos anteriores al caso mexicano, expandieron los servicios bancarios a partir de las bancas provinciales $o$ locales. Tal fue el caso de los llamados joint stocks banks ingleses, o el sistema de bancas provinciales francés y español de la primera mitad del siglo XIX, así como en los numerosos bancos estadunidenses, pertenecientes al régimen de banca libre. Estos establecimientos cumplieron similares funciones a las cubiertas por las instituciones que formaron el primer sistema bancario mexicano (1864-1915). En todos los casos esas primeras experiencias desaparecieron a medida que la regulación monetaria y financiera fue generalizándose a través de las bancas centrales. Así ocurrió en Inglaterra con el Acta Peel en 1844 y en Francia a partir de que fue definido en la orden política imperial de Napoleón III. ${ }^{8}$ Lo mismo

${ }^{8}$ Véase Plessis, Banque, 1982. 
ocurrió en España a partir de $1874^{9}$ y en Estados Unidos con la fundación del Sistema Federal de Reserva en 1913. ${ }^{10}$

La regulación bancaria por parte del gobierno inició tardíamente en México. Los primeros pasos, aún tímidos, fueron definidos en la reforma a las instituciones de crédito de 1908 , lo que fue seguido de la abrupta interrupción de este sistema ordenado por las fuerzas constitucionalistas entre 1913 y 1915, lo cual fue seguido de numerosos cambios y ajustes, lo que concluyó con la fundación del Banco de México y su posterior confirmación en tanto que banca central.

Cabe advertir que el fondo de los Antiguos Bancos de Emisión conserva solamente una parte de los archivos de los bancos de emisión, cuya cancelación ocurrió en diversos momentos, como se observa en los anexos 1 y 2.

Las primeras instituciones de crédito en desaparecer fueron el banco de Campeche que fue declarado en quiebra, los dos bancos yucatecos que se fusionaron en uno solo (Peninsular Yucateco), así como el de Michoacán que se transformó en refaccionario. Tales decisiones ocurrieron en el marco de la crisis financiera que sacudió al gobierno porfiriano durante sus últimos años. Posteriormente, entre 1916 y 1917, se declaró la caducidad a quince bancos de emisión, acusados de rebeldía por la Comisión Reguladora e Inspectora de Instituciones de Crédito, que entre otros objetivos tuvo la tarea de revisar los estados financieros de los bancos. La cancelación de estas instituciones se explicó en razón de haberse negado a mostrar sus libros y el estado de sus cajas, por lo que se consideró que sus operaciones no se ajustaban a los têrminos establecidos por la Ley de Instituciones de Crédito. ${ }^{11}$

La mayoría de estos bancos cerraron sus puertas, en tanto que otros optaron por el camino de la querella judicial para rescatar sus prerrogativas. Otro, como el Banco Oriental de Puebla, aceptó perder su facultad emisora. Durante el gobierno del general Obregón se buscaron soluciones y arreglos con los banqueros, entre ellas destaca la celebración de la primera Convención Nacional Bancaria (1923), así como la orden de devolución de los préstamos obligatorios y decomisos impuestos por las filas

${ }^{9}$ La experiencia de esas firmas en Sudriá, "Bancos", 1994, pp. 81-107. Asimismo en este volumen el texto de Anes, "Banco", 1994, pp. 109-116.

${ }^{10}$ Intrator, Système, 1996, pp. 5-20.

" Éstos fueron el Peninsular de Yucatán, Hidalgo, Guerrero, Querétaro, San Luis Potosí, Coahuila, Oriental de México, Jalisco, Aguascalientes, Guanajuato, Morelos, Durango, Minero de Chihuahua, Tamaulipas y Mercantil de Monterrey. 
constitucionalistas a los bancos. ${ }^{12}$ Se pretendía que con la devolución de los adeudos las instituciones pudieran rehabilitarse y desempeñarse como bancos refaccionarios asociados al Banco de México.

Las promesas gubernamentales no pudieron cumplirse, dada la situación financiera que padecía el país ante la baja de los productos de exportación en el mercado internacional durante los años de la entreguerra. No obstante, fue inaugurado el Banco de México sin poder llenar plenamente sus funciones como banca central, y algunos de los bancos desincautados prestaron servicios como banca refaccionaria, como fueron los de la ciudad de México, los de Monterrey (Mercantil de Monterrey y el de Nuevo León), así como el Occidental de México.

En tanto que el resto de los bancos que no pudieron realizar sus operaciones pasaron a la administración del Comité Liquidador de Bancos, cuyas facultades fueron autorizadas en la ley del 30 de agosto de 1930, emitida durante el periodo de gobierno de Pascual Ortiz Rubio, por la cual se autoriza la liquidación de los antiguos bancos de emisión, cuyos archivos son lo que constituyen estos acervos en el AGN.

\section{III}

El breve recordatorio histórico permite vislumbrar con claridad la importancia que tiene este fondo documental, que abarca cerca de medio siglo de la historia mexicana. El cual, no obstante, es una de las etapas mejor conocidas en sus manifestaciones políticas, pero es pobre y reducido nuestro entendimiento acerca del estado de su economía. Por lo que, en general, continuamos sustentando nuestro conocimiento de estos procesos en las versiones que fueron legadas por actores pertenecientes a las facciones triunfantes, las del constitucionalismo.

En el fondo de los Antiguos Bancos de Emisión en el AGN, el futuro investigador podrá consultar esta rica información en los libros y expedientes, entre los que se encuentran los siguientes.

1) Libros de contabilidad. Estos documentos son la parte más importante del fondo. Se trata de la información contable de cada uno de los establecimientos, pero, desgraciadamente, no son continuas las series. Por lo que su consulta requiere ser completada con otras fuentes estadísticas, como son los balances mensuales y los informes de los intervento-

${ }^{12}$ Éstos fueron los bancos de la ciudad de México, el Nacional de México y el de Londres y México. Además de varios establecimientos estatales: el Mercantil de Veracruz, el Oriental de México, los de Querétaro y de Zacatecas, el Occidental de México, el Peninsular Mexicano y el del Estado de México. 
res que fueron publicados en las Memorias de instituciones de crédito (18971911). ${ }^{13}$ Las pesquisas numéricas se pueden completar también en las Memorias de los secretarios de Hacienda de esos años, así como en registros semanales o mensuales que fueron publicados en la prensa especializada (ElEconomista Mexicano, por ejemplo) o en los diarios nacionales editados en la ciudad de México. La documentación contable comprende:

a) Los libros diarios y libros de caja, en los cuales se registraron, diariamente, todas las operaciones contables de la institución. Se preservan los ejemplares de la casa matriz y de las sucursales. Las colecciones no son completas, pero, para aquellos que tengan interés en reconstruir la contabilidad de estos bancos, será necesario completar la información de los libros diarios y de caja con los libros mayores, en donde se resume mensualmente la información de los anteriores.

b) Los libros mayores y los libros de balance, correspondientes a los registros incorporados a los balances anuales, del pasivo y activo bancario, son una fuente inestimable para la formación de series acerca del funcionamiento cotidiano de los bancos. ${ }^{14}$

2) Documentos útiles para la realizar la reconstrucción de la radiografía social de los accionistas y de los cuentahabientes de los bancos, así como para el reconocimiento de los mercados de crédito "reales", ya que desde el punto de vista formal las instituciones estatales de crédito no podían hacer circular sus billetes más allá de los limites de la jurisdicción de la entidad, pero esto no fue así, como se aprecia a simple vista por la presencia de varias sucursales o agencias fuera de estos espacios. Para este tipo de estudios pueden ser de utilidad diversos tipos de documentos localizados en este fondo, como son:

a) Los libros de acciones, que contienen información sobre los poseedores del capital de los bancos a través del tiempo, lo que permite realizar la radiografía social de las elites económicas y financieras que participaron en estas empresas. Además de constituir un indicador dentro del comportamiento del naciente mercado bursátil mexicano. Esto último se enriquece con la conservación de acciones de otras sociedades agrícolas, mercantiles o manufactureras, que fueron depositadas en las cajas de estos bancos como garantía de algún préstamo.

b) Los libros de acreedores contienen datos relativos al debe y haber de los créditos otorgados por el banco a una amplia clientela, junto con los libros auxiliares donde se registran diversas cuentas dudosas, créditos

${ }^{13}$ La colección completa se encuentra en el Fondo Reservado de la Biblioteca Nacional y en la Biblioteca del Archivo General de la Nación.

${ }^{14}$ Ejemplo de este tipo de trabajo es la tesis de Gómez, "Sistema", 2001. 
concedidos, además de los valores en cartera y los depósitos a cinco días, hipotecas, etcétera.

c) En este sentido también son útiles los libros de certificados, en los cuales se informa de los adeudos de personas físicas y morales, cuyos datos aparecen en esos certificados bancarios. Complemento de estos documentos son los libros de certificados de depósito y certificados de rédito, que contienen listas y/o talonarios de registro de los bienes depositados para garantizar los préstamos otorgados por el banco o los préstamos otorgados por el mismo, donde se especifica el rédito a cubrir.

d) Especial riqueza para el historiador en búsqueda de la reconstrucción de las clientelas y de la definición de los ámbitos de los mercados de crédito son los datos que presentan los libros de cuentas corrientes (fecha y nombre de los acreedores, deudores, créditos hipotecarios y valores en cartera), de cuentas diversas, que contienen las cuentas malas y dudosas, dividendos del banco, así como operaciones con otras instituciones, y los libros de cartera en donde se inscribieron los préstamos a diversos deudores, se anuncian los remates de bienes, o los juicios llevados ante los tribunales, entre otros datos.

e) Además de estos fondos, para algunos bancos hay también los libros de créditos y/o libros de hipotecas (registros de préstamos de corto y de mediano plazo), los libros de depósitos, en los que se muestran los montos $\mathrm{y}$ fechas de vencimiento de los préstamos, y libros especiales de deudores $\mathrm{y}$ acreedores, en operaciones de terceros donde intervinieron los bancos.

f) Algunos de los archivos de bancos cuentan también con los libros de inmuebles, en los cuales se presentan las operaciones bancarias sobre venta y compra de propiedades que están bajo resguardo del banco.

g) Se recomienda revisar los expedientes del rubro "archivo" con información diversa y dispersa como son las escrituras de bienes depositados, planos de minas o de los inmuebles dados en garantía, y una gran variedad de títulos y obligaciones, además de cartas de presentación o de opinión de terceros clientes que daban fe de la probidad de los futuros clientes.

3) Documentos y expedientes útiles al historiador para reconocer y reconstruir la amplitud y funcionamiento de los mercados de crédito en el país y el extranjero.

a) Se trata de libros especiales en los cuales fueron inscritas las operaciones de canje de billetes, créditos, avances y depósitos que estas empresas entablaron con el Banco Central Mexicano, ${ }^{15}$ que fungió como caja de compensación entre las distintas instituciones de crédito del país.

${ }^{15}$ Fundado el 28 de enero de 1899 con un capital de 6000000 de pesos. La representación de su capital fue dividida en dos series de acciones: la serie A, suscrita por el público y la serie B, por bancos de los Estados. El Banco Central sería el corresponsal de los bancos locales que invirtieran 
b) Libros de corresponsales en el país y en el extrajero, donde se registraron las operaciones con numerosas instituciones nacionales y extranjeras, entre las que destacan las operaciones realizadas con el Banco Hispanoamericano.

c) Libros de remesas, en los que se registraron los giros y remesas u órdenes de cobro de cheques con instituciones o personas en el país y en el extranjero.

d) Existen varios tipos de libro de registro y talonarios en los cuales están los generales, que contienen información sobre los siguientes asuntos: giros enviados, registro de escrituras, registro de firmas. Los de registro de billetes, con anotaciones sobre las series, valores, fechas y firmas de los billetes puestos en circulación. Los libros de registro de giros en los que se muestran las fechas, el número y nombre del destinatario del giro.

4) Libros para conocer la historia interna de la empresa bancaria.

a) Para el estudio de la organización y administración interna existen los libros de gastos generales, en los cuales aparecen los pagos de cobro de la luz, del teléfono, de la renta de los inmuebles y del agua, para la sede en la capital de los estados y las sucursales. Los libros de inventario registran los gastos generales del banco y para el mantenimiento del inmueble.

b) Los libros de vales donde hay testimonio de las operaciones del banco con su personal, como era el caso de los adelantos y los préstamos provisionales.

c) De gran valor y utilidad para el estudio de la política y decisiones de los directivos bancarios son los libros del Consejo de Administración y los libros de Asamblea. Los primeros contienen los acuerdos de las juntas de accionistas, las resoluciones para la aprobación de la emisión de billetes, las actas de aprobación del Consejo de Administración del Banco. Y los segundos reúnen las actas de las asambleas ordinarias y extraordinarias que celebraban los accionistas del banco. Estos documentos son muy engañosos, ya que pocos son los consejos directivos que dejaron asentados los criterios de su política o de las decisiones y recomendaciones de la firma. Además, en el transcurso de los años cambia el registro de los acuerdos, dependiendo de la composición del Consejo. De cualquier forma es una fuente indispensable para conocer el funcionamiento del banco.

$5 \%$ de su capital en acciones de la serie $\mathrm{B}$, la cual después de diez años sería convertible en serie $\mathrm{A}$. El Banco Central abrió a cada banco local una cuenta corriente hasta por $10 \%$ de su capital pagado, con intereses de $5 \%$ a favor de los bancos locales y $7.5 \%$ a favor del Banco Central. Si la cuenta mostraba un saldo a favor de un banco local superior a $10 \%$ de su capital pagado, ganaría $8 \%$ sobre el saldo, pero pagaría 9.5\% al Central si su saldo disminuyera de ese $10 \%$.

El Banco Central redimiría a la par los billetes de los bancos locales, hasta un total igual al monto de su crédito. Otras operaciones bancarias de carácter mutuo fueron también estipuladas. 


\section{IV}

Trunca quedaría esta exposición sobre los antiguos bancos de emisión, si no hacemos referencia a otro tipo de acervos documentales y bibliográficos, de obligada revisión a la hora de realizar un estudio sobre estos establecimientos o sobre las elites y el mercado financiero durante las primeras tres décadas del pasado siglo $\mathrm{Xx}$, como es el caso de los archivos históricos de BanameX y el del Banco de México. ${ }^{16}$

Otras fuentes de archivo de gran utilidad para la comprensión y estudio de la banca de emisión porfiriana son los archivos personales de José Ives Limantour y de Miguel Montes de Oca que resguarda el Centro de Estudios Históricos de CONDUMEX, pertenecientes a los antiguos secretarios de Hacienda del periodo porfirista y posrevolucionario.

A los futuros investigadores les recomiendo también revisar diversos fondos documentales en el AGN, como aquellos que se localizan en el ramo de Presidentes al grupo documental Obregón/Calles, que contiene material sobre la Comisión Nacional Bancaria, la reorganización bancaria, la crisis económica, el Banco de México, además de información sobre el Banco de Londres y México y los bancos provinciales de Sonora, Tamaulipas, Occidental de México, Oriental de México y Minero de Chihuahua. Asimismo, el ramo de Presidente Pascual Ortiz Rubio, en él hay varios documentos sobre el Banco de Londres y México referentes a la probable quiebra del establecimiento, además de invitaciones, informes y discursos con motivo del 50 aniversario del Banco de Nuevo León.

Entre las fuentes impresas de obligada consulta, por ser complemento de los archivos, sugiero la revisión de los capítulos sobre instituciones de créditos que contienen la veintena de volúmenes publicados con el título de Memorias de Hacienda. Así como los tomos de las Memorias de Instituciones de Crédito, publicadas entre 1900 y 1911, y los artículos y datos difundidos tanto por la prensa especializada, en especial El Economista Mexicano, como por los cotidianos, que incluyen en sus últimas páginas información de los establecimientos bancarios más importantes, en particular los balances anuales o mensuales de éstos.

Los archivos bancarios que se preservan en el fondo de Antiguos Bancos de Emisión del AGN son una fuente de consulta relevante para los futuros historiadores. Cabe advertir que estos materiales no sólo intere-

La más importante consistió en la formación de un fondo especial para ayuda de los bancos del sistema que pudieran encontrarse en condiciones aflictivas, por medio de una contribución no mayor de $2 \%$ del capital de cada banco.

${ }^{16}$ El archivo no está abierto al público, no obstante es meritorio el esfuerzo y los resultados alcanzados después de largos años de trabajo, que iniciaron gracias al impulso de Francisco Borja, además del cuidado y atención que en ello ha prestado Eduardo Turrent. 
san a los estudiosos de los fenómenos crediticios y monetarios. También son fundamentales para otros campos de la historiografía mexicana, en un periodo clave como fueron las primeras décadas del siglo $\mathrm{xx}$, durante las cuales transcurre el declive del porfiriato, la lucha armada y los primeros años de la reordenación económica llevada a cabo por los gobiernos posrevolucionarios. Además de que el carácter estatal de estas empresas hace de sus archivos una fuente clave para el enriquecimiento de la historia regional, óptica que ha estado en boga en los últimos años, enriqueciendo el conocimiento y comprensión del pasado mexicano.

Esto último se aprecia en la diversidad de temas y problemas de investigación que pueden ser fundamentados en estos fondos archivísticos. A manera de ejemplo se sugiere:

1) La historia bancaria y de los mercados de crédito en el ámbito nacional y regional, ${ }^{17}$ que puede ser analizada a partir de los libros con los corresponsales nacionales y en particular con el Banco Central.

2) La historia de las elites y de las redes de poder, que se observa en los accionistas y en los diversos registros de las cuentas de los clientes.

3) La historia financiera internacional, dados los vínculos entre los bancos provinciales y los corresponsales en Europa y Estados Unidos, fundamentalmente.

4) La historia local y regional, ya que además de tratarse de establecimientos con jurisdicción estatal, existe una abundante información acerca de las operaciones en sus sucursales y agencias. ${ }^{18}$

5) La historia laboral y de la administración bancaria, a partir de los registros sobre empleados, de sus salarios y prestaciones que aparecen registrados en estos documentos. ${ }^{19}$

A manera de conclusión, considero que la conservación de los archivos de empresa, y entre ellos los de bancos, forman parte de la cultura histórica de un país, que no es otra cosa sino el modo y la forma como una colectividad o sociedad se relacionan con su pasado. Lo que se manifiesta de diversas formas, que van desde la memoria histórica en el discurso político hasta el reconocimiento social que han alcanzado los profesionistas

\footnotetext{
${ }^{17}$ Recientemente fue publicado bajo la coordinación de Marichal y Cerutti, una compilación de diversas monografías sobre los bancos estatales. Marichal y Cerutti, Banca, 2003.

${ }^{18}$ El primer estudio de historia local bancaria realizado con estos fondos ha sido elaborado por Ordóñez, "Sucursal", 2002.

${ }^{19}$ El primer trabajo acerca de la historia de los empleados bancarios ha sido incluido en la tesis de Lorenzo, "Banqueros", 2000.
} 
de la historia, así como el impacto intelectual de la creación histórica en las manifestaciones culturales. Además, de lo que nos ocupa en este artículo, es decir, la atención y cuidado que se tiene por parte de las autoridades públicas o de grupos privados para conservar y enriquecer los fondos de obras históricas y de los archivos. ${ }^{20}$

Son invaluables los avances logrados en la organización de acervos documentales de algunos bancos. Pero éstos son muy reducidos. Espero que iniciativas como ésta se multipliquen en el futuro y no se vean interrumpidos por cambios administrativos, siendo, además, recomendable que tales iniciativas se contagien a otras instituciones para que permitan en forma permanente la consulta de sus archivos a los estudios.

De acuerdo con lo anterior podemos reconocer que la cultura histórica mexicana, el aprecio por el pasado es una tradición rica y sólida frente a la conservación de los edificios y monumentos prehispánicos y coloniales. Pero el patrimonio histórico de una nación debe ser prolongado en el tiempo incorporando al siglo $\mathrm{xx}$, y en él deben ingresar otros sujetos históricos relevantes como es el caso de las empresas, y de los bancos que fueron parte relevante del desarrollo social y económico del México moderno y contemporáneo. Se requiere evitar su destrucción a fin de reconocer el peso de los servicios bancarios en la transformación del México moderno. Más allá de consideraciones a favor y en contra de estas empresas, sobre las cuales se mantienen añejos prejuicios y fundados reclamos en la época presente, considero que la conservación de los archivos bancarios por parte de sus directivos o de las autoridades públicas o incluso por iniciativa académica es un requerimiento previo para que el futuro trabajo del historiador pueda ser realizado libre de conjeturas, prejuicios y reclamos sin fundamento.

Razón por la cual, considero que se requiere de la definición de políticas definidas para resguardar la memoria histórica en los documentos y archivos de diversas firmas y sociedades anónimas. Es necesario reaccionar antes de que sean destruidos o abandonados por falta de cuidado y conservación, ante la poca atención de las autoridades oficiales.

\section{BIBLIOGRAFIA}

Anes Álvarez, Rafael, "El Banco de España, la deuda pública y la política monetaria entre 1874 y 1918 ” en PEDro TEDde y CARLOS Marichal, La formación central en España y América Latina (siglos XIX y XX), Madrid, Banco de EspañaServicio de Estudios, 1994, pp. 109-116 (Estudios de Historia Económica, 29).

${ }^{20}$ Le Goff, Histoire, 1988, pp. 218-219. 
Archivo General de la Nación, Guía general Juan Manuel Herrera y Victoria San Vicente (coords.), presentación Leonor Ortiz Monasterio, México, núm. 224, 1990.

GÓMEZ, MONICA IRENE, "Un sistema bancario con emisión de billetes por empresarios privados. El comportamiento del Banco Nacional de México en el proceso de creación de dinero en México, 1884-1910", tesis de doctorado en Historia, CEH-El Colegio de México, 2001.

INTRATOR, HÉlÈne, Le système bancaire américain, París, Presses Universitaires de France, 1996, pp. 5-20 (Que sais-je?, 3171).

Le Goff, JACQUeS, Histoire et mémoire, París, Gallimard, 1988 (Folio-Histoire).

LoRENZO, MARfA Dolores, "Banqueros y empleados españoles en Puebla, Oaxaca y Chiapas. El Banco Oriental de México, 1900-1915”, tesis de maestría en Historia de México, Facultad de Filosofía y Letras-UNAM, 2000.

LUDLOW, LEONOR, "La primera etapa de formación bancaria (1864-1897)" en LEONOR LUDLOW y JORGE SILVA-RIQUER (comps.), Los negocios y las ganancias de la colonia al México Moderno, México, Instituto Mora/Instituto de Investigaciones Históricas-UNAM, 1993.

(coord.), Catálogo de fondos documentales del Banco Oriental de México, 1900-1932, México, Instituto Mora, 2001 (Perfiles-Fuentes).

"Ejemplos de instituciones bancarias (siglos XVIII y XIX)" en VIRGINIA GUEDEA y LEONOR LUDLOW (coords.), El historiador frente a la historia. La historia económica, México, Instituto de Investigaciones Históricas-UNAM, 2003. - y CARMen Blázquez (coords.), Catálogo de fondos documentales del Banco Mercantil de Veracruz 1897-1933, México, Instituto Mora, 1997 (PerfilesFuentes).

Marichal, Carlos y Mario CerutTi (coords.), La banca regional en México (18701930), México, Fondo de Cultura Económica/El Colegio de México, 2003.

ORdóñzZ LOPEZ, CÉSAR AuguSTO, "La sucursal del Banco Mercantil de Veracruz en Xalapa (1904-1910)", tesis de licenciatura en Historia, Universidad Veracruzana, 2002.

Plessis, Alain, La banque de France et ses deux cents actionnaires sous le Second Empire, Ginebra, Librairie Droz, 1982.

SOMBART, Werner, El apogeo del capitalismo, México, Fondo de Cultura Económica, vol. I, 1984.

SUdRiá Triay, Carlos, "Los bancos de emisión provinciales en la España del siglo XIX" en PEDRo TEDde y Carlos MARIChal, La formación central en España y América Latina (siglos XIX y XX), Madrid, Banco de España-Servicio de Estudios, 1994, pp. 81-107 (Estudios de Historia Económica, 29). 


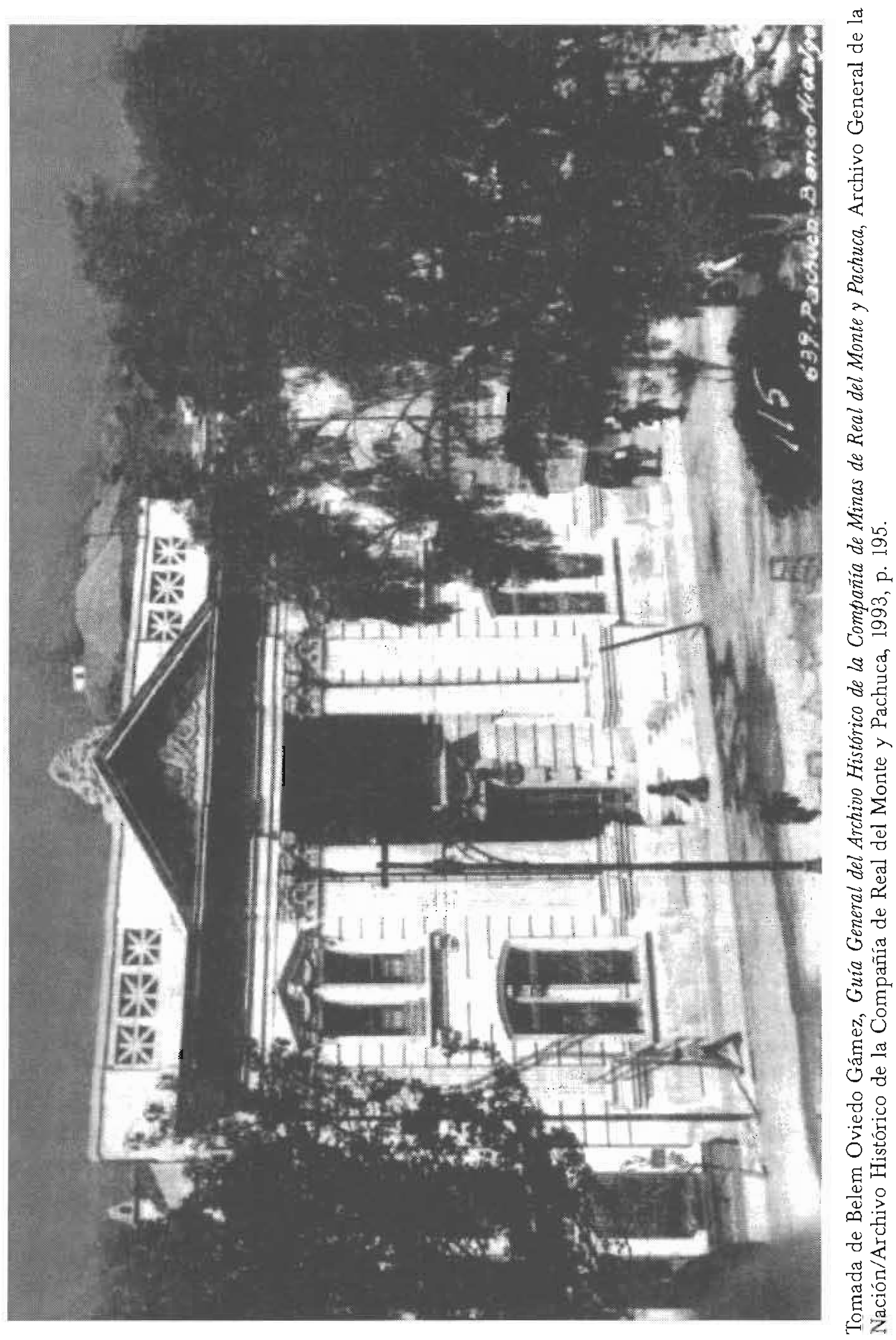

